2004

\title{
Laser-induced fluorescence measurements of three plasma species with a tunable diode laser
}

Amy M. Keesee

Earl E. Scime

Robert F. Boivin

Follow this and additional works at: https://researchrepository.wvu.edu/faculty_publications

\section{Digital Commons Citation}

Keesee, Amy M.; Scime, Earl E.; and Boivin, Robert F., "Laser-induced fluorescence measurements of three plasma species with a tunable diode laser" (2004). Faculty Scholarship. 211.

https://researchrepository.wvu.edu/faculty_publications/211 


\title{
Laser-induced fluorescence measurements of three plasma species with a tunable diode laser
}

\author{
Amy M. Keesee and Earl E. Scime \\ Department of Physics, West Virginia University, Morgantown, West Virginia 26506-6315 \\ Robert F. Boivin \\ Department of Physics, Auburn University, Aubum, Alabama 36849-5311
}

(Presented on 21 April 2004; published 12 October 2004)

\begin{abstract}
Recently, we demonstrated that a single, tunable, low-power, diode laser can be used for laser-induced fluorescence (LIF) measurements of both argon ions and helium neutrals. We have now identified a third fluorescence scheme, for neutral argon atoms, accessible with the same tunable diode laser. Fluorescence measurements of a heated iodine cell are used to monitor the wavelength of the laser during the LIF measurement. () 2004 American Institute of Physics.

[DOI: $10.1063 / 1.1787166]$
\end{abstract}

\section{INTRODUCTION}

In this work, we show that a single low-power, diode laser $^{1}$ can be used to perform laser-induced fluorescence (LIF) measurements on neutral argon atoms, argon ions, and helium atoms. By encompassing both ion and neutral plasma species with a single diode laser, the diode laser can be a powerful diagnostic tool for a wide variety of lowtemperature plasmas. With a viable LIF scheme for neutral argon and neutral helium, it is now possible to directly measure the distribution of neutral atoms in most helicon source experiments.

\section{EXPERIMENTAL APPARATUS}

The hot helicon experiment (HELIX) vacuum chamber is a $61 \mathrm{~cm}$ long, Pyrex tube $10 \mathrm{~cm}$ in diameter connected to a $91 \mathrm{~cm}$ long, $15 \mathrm{~cm}$ diameter, stainless-steel chamber. The chamber has one set of four 6 in. Conflat ${ }^{\mathrm{TM}}$ crossing ports in the center of the chamber and two sets of four 23/4 in. Conflat ${ }^{\mathrm{TM}}$ crossing ports on either side that are used for LIF diagnostic access. The opposite end of the chamber opens into a $2 \mathrm{~m}$ diameter space chamber, the large experiment on instabilities and anisotropies. ${ }^{2}$ Ten electromagnets produce a steady-state axial magnetic field of $0-1200 \mathrm{G}$ in the source. A MKS mass flow controller ${ }^{3}$ is used to introduce gas into the vacuum chamber. Neutral pressures from 0.1 to 100 mTorr are achievable. rf power of up to $2.0 \mathrm{~kW}$ over a frequency range of $6-18 \mathrm{MHz}$ is coupled into a $19 \mathrm{~cm}$ halfwave right-handed helix antenna and creates the steady-state plasma. Characteristic electron temperature and densities in HELIX are $T_{e} \approx 4 \mathrm{eV}$ and $n \geqslant 1 \times 10^{13} \mathrm{~cm}^{3}$ as measured with a rf compensated Langmuir probe. ${ }^{4}$

Our original $\leqslant 15 \mathrm{~mW}, 1.5 \mathrm{MHz}$ bandwidth, Sacher Lasertechnik SAL-665-10 diode laser was mounted in a Littrow external cavity and had a mode-hop free tuning range of up to $14 \mathrm{GHz}(\approx 0.021 \mathrm{~nm}) .{ }^{1}$ We recently upgraded our diode

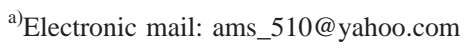

laser with another Littrow mounted diode, a SAL-665-20, with a mode-hop free tuning range of $12 \mathrm{GHz}$ and an increased output power of $\leqslant 20 \mathrm{~mW}$. Wavelength scanning is accomplished by varying the voltage on a piezoelectric controlled grating located within the laser cavity. ${ }^{5}$ A National Instruments input/output card provides the voltage ramp to scan the laser frequency and the linearly polarized laser is mounted on a vibration-isolated platform. The laser light is directed through an iodine cell and into the plasma with a series of mirrors and beam splitters mounted on the vacuum chamber and the laser table. The optical configuration is described in detail in Ref. 1. A tightly focused set of optics, mounted on a radially scanning assembly, collects the fluorescence light perpendicular to the injected beam and sends the light through a fiber optic cable to a filtered $(1 \mathrm{~nm}$ wide bandpass) Hamamatsu infrared detector. ${ }^{6}$ The detector signal is composed of fluorescence radiation, electron-impactinduced radiation, and electronic noise. A mechanical chopper operating at a few $\mathrm{kHz}$ is used to modulate the laser beam before it enters the vacuum chamber and a Stanford Research Systems SR830 lock-in amplifier ${ }^{7}$ is used to eliminate all noncorrelated signals. Lock-in amplification is indispensable since the electron-impact-induced emission is several orders of magnitude larger than the fluorescence signal. The laser wavelength is tuned to the appropriate transition with a Burleigh WA-1500 wavemeter ${ }^{8}$ and monitored for frequency drift with a simultaneous molecular iodine fluorescence measurement during each scan of the laser frequency.

Because the weak absorption lines of molecular iodine in the wavelength range of all three LIF schemes are difficult to fluoresce in commercially available iodine cells, a modified iodine cell is required for use with our low-power diode laser. Increased vapor pressure of molecular iodine in the reference cell can be obtained with either a custom iodine cell containing a larger than normal quantity of crystalline iodine, ${ }^{9}$ or by heating a commercially available iodine cell. We chose to construct a thermostatically controlled heater for our $100 \mathrm{~mm}$ long, $25 \mathrm{~mm}$ diameter, Opthos Instruments iodine cell. ${ }^{10}$ Watlow flexible silicone rubber heaters ${ }^{11}$ were 


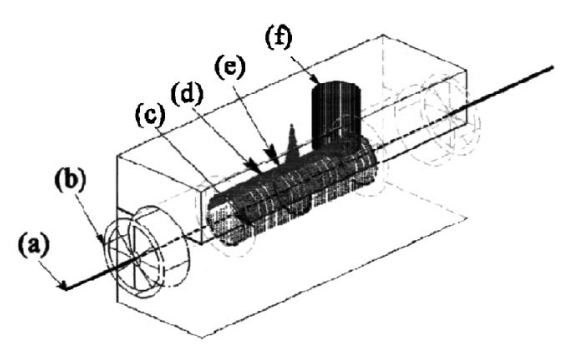

FIG. 1. Housing for the heated iodine cell: (a) Laser beam, (b) iris diaphragm, (c) flexible silicone heaters, (d) thermocouple, (e) iodine cell, and (f) photodiode detector.

attached to the cell and the heated cell placed in a thick walled aluminum housing (see Fig. 1). The Watlow heaters are controlled by an Omega CNi3252 process controller using a $K$-type thermocouple for temperature monitoring. ${ }^{12}$ Oriel iris diaphragms ${ }^{13}$ were mounted on each end of the housing to limit ambient light while allowing the laser beam to pass through the cell. Fluorescent emission from the molecular iodine vapor is detected with a battery powered, UDT Sensors PIN photodiode ${ }^{14}$ and amplified with a Stanford Research Systems SR560 Preamplifler. ${ }^{7}$ For an incident laser power of $17 \mathrm{~mW}$, excellent signal to noise for the molecular iodine fluorescence measurement was obtained for an iodine cell temperature of $69^{\circ} \mathrm{C}$.

\section{LASER-INDUCED FLUORESCENCE MEASUREMENTS}

Complete details of the argon ion ${ }^{15}$ and helium neutral LIF $^{1}$ schemes used with our low power, tunable, diode laser have been published previously. The level sequences are shown in Fig. 2. The Ar-II LIF scheme is a three-level sequence that begins with the $3 d^{4} F_{7 / 2}$ metastable state. The He-I LIF scheme is a four-level scheme that requires an electron-impact collisional excitation transfer for fluorescence at a wavelength different from the pump wavelength. The Ar-I LIF sequence we have developed [Fig. 2(c)] has a similar requirement for a significant level of electron collisions with neutral argon atoms.

Our three-level LIF scheme for Ar-I uses laser emission at $667.9125 \mathrm{~nm}$ (in a vacuum) to pump the $4 s\left({ }^{2} P_{3 / 2}^{0}\right)_{1}$ state to the $4 p^{\prime}\left({ }^{2} P^{0}{ }_{1 / 2}\right)_{0}$ state $\left(1 s_{4}\right.$ to $2 p_{1}$ in Paschen notation), which then decays to the $4 s^{\prime}\left({ }^{2} P^{0}{ }_{1 / 2}\right)_{1}$ state $\left(1 s_{2}\right)$ emitting a photon at $750.5934 \mathrm{~nm}$. While the $4 s\left({ }^{2} P^{0}{ }_{3 / 2}\right)_{1}$ state is not a ground or metastable state, we expected a sufficient population for LIF due to direct excitation from the ground state and electron-impact excitation transfers from nearby metastable states $4 s\left({ }^{2} P_{3 / 2}^{0}\right)_{2}$ and $4 s^{\prime}\left({ }^{2} P^{0}{ }_{1 / 2}\right)_{0}\left(1 s_{5}\right.$ and $1 s_{3}$, respectively). For Ar-I, $j j$-coupling must be used to describe the
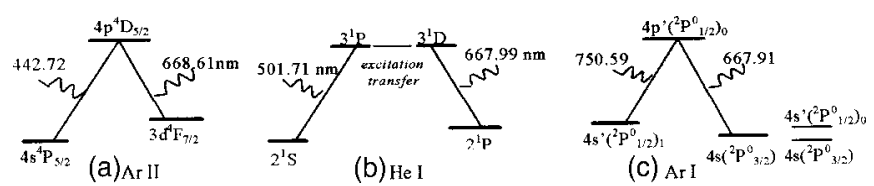

FIG. 2. LIF schemes for (a) Ar-II, (b) He-I, and (c) Ar-I. All wavelengths are in vacuum. The nearby metastable states are also shown for the initial state of the Ar-I sequence. The vertical arrow indicates increasing energy of the atomic states.

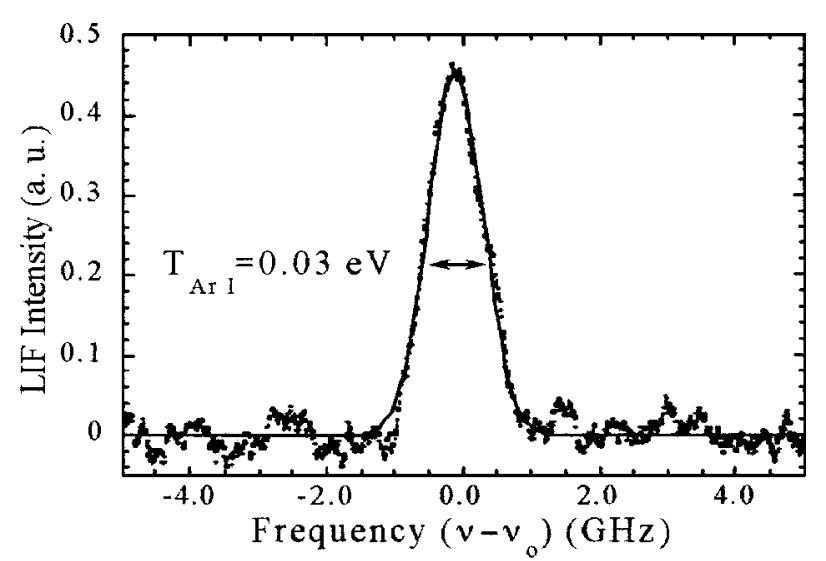

FIG. 3. Typical Ar-I LIF signal vs laser frequency and fit (solid line).

sublevels because the interaction between the spin of each electron and its own orbit is greater than the interactions between the two spins and the two orbits. ${ }^{16}$

To obtain reasonable Ar-I LIF signal to noise, the source was operated at pressures above 6.5 mTorr. Optimal signal to noise was achieved for neutral pressures of approximately 10 mTorr. These relatively high neutral pressures provide the collision rates necessary to populate the initial $4 s\left({ }^{2} P^{0}{ }_{3 / 2}\right)_{1}$ state. A typical Ar-I LIF measurement is shown in Fig. 3. The simultaneously acquired molecular iodine fluorescence spectrum is shown in Fig. 4(c). Although other groups have performed LIF measurements on neutral argon, ${ }^{17}$ to the best of our knowledge, this is the first use of this three-level sequence for neutral argon.

For typical helicon source argon plasma parameters, the total absorption line shape (LIF lineshape) is a convolution of thermal (Doppler) broadening and Zeeman splitting. Other effects, such as the natural linewidth of the line, the laser linewidth, and Stark broadening, are ignorable. For the specific Ar-I transition used in this work, the Zeeman splitting yields three components. ${ }^{16}$ For the $\pi$ transition, the magnetic orbital quantum number for each level is the same $(\Delta M$ $=0$ ). This transition is unshifted from the central wavelength and is linearly polarized along the magnetic field. For the two circularly polarized $\sigma$ transitions, the magnetic orbital quantum number for each level is different $(\Delta M= \pm 1)$. The line shifts for the $\sigma \pm$ transitions in a $1 \mathrm{kG}$ magnetic field are $\Delta \lambda= \pm 2.08 \times 10^{-2} \AA .{ }^{18}$ Relative intensities of all three Zeeman components obey $I_{\pi}=2 I_{\sigma}$.

When the polarization axis of the laser is oriented parallel to the axial magnetic field (perpendicular laser injection), only the $\pi$ transition is pumped. For parallel laser injection, both of the $\sigma$ transitions are pumped. Insertion of a quarterwavelength retarder in the laser path creates circularly polarized light and enables pumping of a single $\sigma$ transition for parallel laser injection. Because only a single transition is pumped for both parallel and perpendicular laser injection, all Ar-I LIF measurements can be fit with a single Maxwellian distribution shifted by the Zeeman splitting (for the $\sigma$ lines) and the bulk flow of the neutral atoms: ${ }^{19}$ 


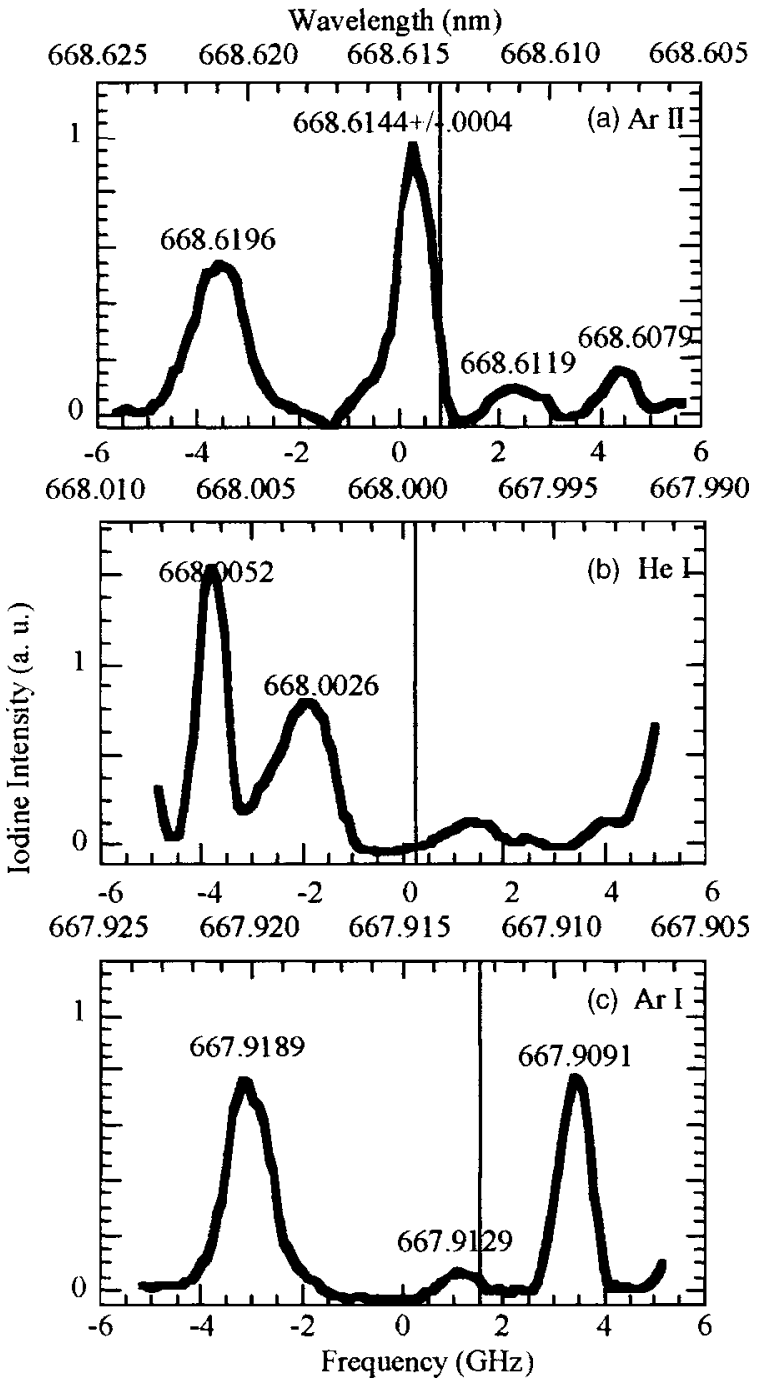

FIG. 4. Intensity vs wavelength and frequency shift of iodine cell fluorescence near the pump wavelength of the LIF scheme for (a) Ar-II, (b) H-I, and (c) Ar-I. The laser pump wavelength is indicated with a vertical line for each species.

$$
I_{R}(\nu)=I_{R}\left(\nu_{0}\right) \exp \left[\frac{-\left(\nu-\Delta \nu_{z}-\nu_{0}-V_{0} \nu_{0} / c\right)^{2}}{\alpha_{0} T_{n}}\right],
$$

where $I_{R}(\nu)$ is the measured LIF signal, $\Delta \nu_{Z}$ is the Zeeman shift relative to the rest frame transition frequency $\nu_{0}\left(\Delta \nu_{Z}\right.$ $=\varepsilon B ; \varepsilon= \pm 1.4 \mathrm{GHz} / \mathrm{kG}$ for the $\sigma$ lines; $\varepsilon=0$ for the $\pi$ transition; and $B$ is the magnetic field in $\mathrm{kG}),\left(V_{0} \nu_{0} / c\right)$ is the overall Doppler shift of the distribution due to bulk flow of the neutrals, $\alpha_{D}$ scales the width of the thermal broadening for neutral argon of mass $m_{n}\left(\alpha_{D}=2 k \nu_{0} / m_{n} c^{2}\right)$, and $T_{n}$ is the temperature of the neutrals in eV. A fit of Eq. (1) to the Ar-I $\pi$ transition LIF measurement shown in Fig. 3 yields a neutral temperature of $0.03 \mathrm{eV}$. Given the relatively high neutral pressures required for the LIF measurement, a neutral temperature consistent with temperature of the chamber walls, $\sim 0.03 \mathrm{eV}$, is not surprising.

The shift of the measured velocity distribution relative to the iodine cell fluorescence lines is used to determine the bulk flow the interrogated species for all three LIF schemes. The absolute wavelengths and relative intensities of the molecular iodine fluorescence for a $12 \mathrm{GHz}$ wide scan in the vicinity of the central absorption wavelength of each of the three different LIF schemes is shown in Fig. 4. The absolute wavelengths of each fluorescence peak were determined with the Burleigh wavemeter (with an absolute accuracy of $\pm 0.00003 \mathrm{~nm}$ according to the manufacture ${ }^{20}$ and an observed statistical uncertainty of $\pm .0004 \mathrm{~nm}$ ). The temperature of the heated iodine cell was kept at $69.3 \pm 0.4^{\circ} \mathrm{C}$. None of these very weak iodine peaks (all of Fig. 4) appear in the standard iodine tables. ${ }^{21}$ The iodine lines for the Ar-II sequence [Fig. 4(a)] are consistent with the weakest of the iodine lines reported in Fig. 3 of Severn et al..$^{15}$ is original Ar-II LIF work. The spacings in GHz between the LIF peak and the nearest iodine cell peak are $-0.40,-2.02$, and +2.28 for Ar-II, He-I, and Ar-I, respectively.

We have shown that LIF can be performed on argon neutrals with the same compact, low-power, tunable diode laser capable of performing LIF on argon ions and helium neutrals. The measured iodine cell fluorescence spectrum in a narrow frequency range around all three LIF schemes is too weak to appear in the published iodine cell absorption tables and is provided here as an aid to future LIF experimenters.

\section{ACKNOWLEDGMENTS}

This work was performed with support from the U.S. Department of Energy under Grant No. DE-FGO297ER54420 and one of the authors (A.M.K.) was supported by a Department of Energy Fusion Energy Sciences Fellowship.

${ }^{1}$ R. F. Boivin and E. E. Scime, Rev. Sci. Instrum. 74, 4352 (2003).

${ }^{2}$ E. E. Scime et al., Phys. Plasmas 7, 2157 (2000).

${ }^{3}$ MKS Instruments, Inc., 6 Shattuck Rd., Andover, MA 01810-2449.

${ }^{4}$ I. D. Sudit and F. F. Chen, Plasma Sources Sci. Technol. 3, 162 (1994).

${ }^{5}$ Sacher Lasertechnik LLC, Instruction Manual, Hannah Arendt Str. 3-7 D35037 Marburg/Lahn Germany (2000)

${ }^{6}$ Hamamatsu Corporation, 360 Foothill Road, Bridgewater, NJ 08807.

${ }^{7}$ Stanford Research Systems, Inc., 1290-D Reamwood Ave., Sunnyvale, CA 94089.

${ }^{8}$ Burleigh Instruments, Burleigh Park, P.O. Box E, Fishers, NY 14453.

${ }^{9}$ G. Severn and R. McWilliams (personal communication).

${ }^{10}$ Opthos Instruments Inc., 17805 Caddy Drive, Rockville, MD 20855.

${ }^{11}$ Watlow Electric Manufacturing Company, 12001 Lackland Road, St. Louis, MO 63146.

${ }^{12}$ OMEGA Engineering, Inc., One Omega Drive, Stamford, CT 06907.

${ }^{13}$ Oriel Instruments, 250 Long Beach Blvd., Stramford, CT 06497.

${ }^{14}$ UDT Sensors, Inc., 12525 Chadron Avenue, Hawthorne, CA 90250.

${ }^{15}$ G. D. Severn et al., Rev. Sci. Instrum. 69, 10 (1998).

${ }^{16}$ L. Pauling and S. Goudsmit, The Structure of Line Spectra (McGraw-Hill, New York, 1930).

${ }^{17}$ B. K. McMillin and M. R. Zachariah, J. Appl. Phys. 79, 77 (1996).

${ }^{18}$ G. Marr, Plasma Spectroscopy (Elsevier, New York, 1968).

${ }^{19}$ R. F. Boivin, West Virginia University Plasma Physics Lab Report PL050, EPAPS-E-PHPAEN-10-003306, 2003.

${ }^{20}$ Burleigh WA-1500 Operating Manual, Burleigh Instruments, Burleigh Park, P.O. Box E, Fishers, NY 14453 (1998).

${ }^{21}$ S. Gerstenkorn and P. LAS, Atlas Du Spectre D'absorption De La Molecule D'iode (Editions DuCentre National De La Recherche Scientifique, Orsay, France, 1978). 